\title{
Abstract Selection
}

Elective neck dissection in the treatment of T3/T4 N0 squamous cell carcinoma of the larynx. Kligerman, J., Olivatto, L. O., Lima, R. A., Freitas, E. Q., Soares, J. R., Dias, F. L., Melo, L. E., Sa, G. M., Duccini, E. Head and Neck Service, Hospital do Cancer/ INCa., Rio de Janeiro, Brazil. American Journal of Surgery (1995) November, Vol. 170 (5), pp: 436-9.

BACKGROUND: This study analyzed pathologic findings of clinically occult cervical lymph nodes of T3/T4 N0 squamous cell laryngeal carcinoma and their impact on locoregional failures and overall survival. PATIENTS AND METHODS: A retrospective analysis of 76 patients with T3/T4 N0 laryngeal carcinoma was carried out between 1981 and 1989. Sixty-seven patients had transglottic tumour. 31 patients had extralaryngeal spread, 56 patients were T3 N0, and 20 patients were T4 N0. Seventy-five patients had total laryngectomy and one had near total laryngectomy. All patients had bilateral elective neck dissection. The chisquare test was applied to factors related to neck metastasis and locoregional failure. Survival was analyzed using the KaplanMeier actuarial method; differences were tested using the Wilcoxon signed-ranked test. RESULTS: Eighteen patients had positive surgical margins. Occult neck metastasis was observed in 30 per cent. Univariate analysis showed that cancer stage and cartilage status were not significant to predict neck metastasis Locoregional recurrence was observed in 28 per cent of patients. Surgical margins, cervical metastasis, lesion extension, and cartilage invasion had significant impact disease-free survival. The five-year overall survival was 52 per cent; disease-free survival was 57 per cent. CONCLUSION: The elective bilateral neck dissection performed in T3/T4 N0 patients yielded a 30 per cent incidence of occult neck metastasis Classification of transglottic carcinomas into endolaryngeal and exolaryngeal provides a better parameter for predicting neck metastasis than does $T$ status Disease-free and overall survival were significantly affected by neck metastasis $T$ stage, exolaryngeal tumour, cartilage infiltration, and surgical margins. Author.

Factors influencing contralateral metastasis and prognosis from pyriform sinus carcinoma. Kowalski, L. P., Santos, C. R., Magrin, J., Scopel, A. Department of Head and Neck Surgery, Hospital A. C. Camargo, Fundaccao Antonio Prudente, Sao Paulo, Brazil American Journal of Surgery (1995) November, Vol. 170 (5), pp: $440-5$.

BACKGROUND: The presence of neck metastasis reduces the probability of regional control and survival. The purpose of this study is to identify risk factors for contralateral neck metastasis in a series of 218 patients with pyriform sinus carcinoma. PATIENTS AND METHODS: Tumour stages were: 31 T1-2, 187 T3-4, 40 N0, 174 N1-3, and 4 NX. A total of 182 patients ( 83 per cent) had metastatic lymph nodes ( 23 in the contralateral neck). RESULTS: Thirty-five patients presented neck recurrences (27 in contralateral undissected neck). Logistic regression identified $\mathrm{T}$ and $\mathrm{N}$ stages, epilarynx and posterior pharyngeal wall involvement hemilarynx fixation, and ipsilateral level 1 metastasis as important predictors of contralateral metastasis Five-year survival rates were 41 per cent (pN0), 23 per cent (contralateral pN0), and 31 per cent (contralateral positive nodes). CONCLUSIONS: The contralat eral side of the neck was the most common site of recurrence. A lateral neck dissection is advisable for the contralateral side of the neck for high-risk patients Author.

Parotid and neck metastases from cutaneous squamous cell carcinoma of the head and neck. Khurana, V. G., Mentis, D. H., O'Brien, C. J., Hurst, T. L., Stevens, G. N., Packham, N. A Department of Head and Neck Surgery, Royal Prince Alfred Hospital, Sydney, Australia. American Journal of Surgery (1995) November, Vol. 170 (5), pp: 446-50.
BACKGROUND: Approximately five per cent of cutaneous squamous cell carcinomas (SCC) metastasize to regional nodes. Nodal involvement may be more common for cutaneous squamous cell carcinomas of the head and neck, but few large series have reported clinical outcome after treatment of parotid gland and neck metastases from these cancers. PATIENTS AND METHODS: A combined retrospective/prospective study of patients treated between 1983 and 1994 was performed. There were 69 men and six women, with a median age of 67 years. Twenty-nine patients had neck metastases, and 33 had parotid gland metastases, while 13 patients had involvement at both sites Of the 75 patients, 68 were treated surgically and 50 received postoperative radiotherapy. RESULTS: The facial nerve was sacrificed totally in six patients and partially in nine. Histologic extranodal spread was present in 48 (71 per cent) of all surgically treated patients Among 61 patients followed up to recurrence, or for greater than 12 months, 26 ( 43 per cent) developed recurrence-12 in the parotid gland, seven in the neck, and seven in both sites Multiple recurrences were common and occurred at a median of eight months after surgery. Positive surgical margins were associated with poorer local disease control $(P<0.05)$. Cumulative survival at five years was 61 per cent, but only 15 of 70 evaluable patients ( 23 per cent) were eligible for follow-up at this time. Neck involvement with or without parotid gland disease was associated with an increased risk of distant metastases, but this was not statistically significant. Post-operative radiotherapy was not associated with improved disease control. CONCLUSION: Cutaneous, metastatic SCC involving the parotid gland and neck is an aggressive disease with a tendency to an infiltrative growth pattern and multiple recurrences. More aggressive surgery may be justified to reduce the incidence of regional failure after parotidectomy and neck dissection. Author

Prospective study using skin staplers in head and neck surgery. dos Santos, L. R., Freitas, C. A., Hojaij, F. C.. Araujo-Filho, V. J. Cernea, C. R., Brandao, L. G., Ferraz, A. R. Department of Head and Neck Surgery, Hospital das Clinicas, University of Sao Paulo Medical School, Brazil. American Journal of Surgery (1995) November, Vol. 170 (5), pp: 451-2.

BACKGROUND: A prospective trial was undertaken to investigate the advantages and disadvantages of stapled skin closure versus conventional nylon sutures in head and neck surgery. PATIENTS AND METHODS: The study included 20 consecutive patients who underwent extensive surgery in which their skin was closed with staples. Another group of 20 matched patients receiving a non-continuous nylon suture closure was followed in parallel. RESULTS: The complications recorded occurred in five patients in the stapled group and three in the sutured group. Analysis of cosmetic results showed 16 patients ( 80 per cent) in the stapled group with good wound appearance and 17 ( 85 per cent) in the sutured group. The mean closure time was five minutes for the stapled group and 25 minutes for the sutured group. Cost was $\$ 19.75$ for conventional closure and $\$ 22.00$ for mechanical suture. CONCLUSION: The use of skin staples speeds up closure time by 80 per cent, yields similar cosmetic results with no increase in complications, although at a slightly higher cost. Author.

Surgical rescue of recurrent carcinoma of the nasal columella. Mignogna, F. V., Garay, K. F. Head and Neck Surgical Associates Englewood, New Jersey, USA. American Journal of Surgery (1995) November, Vol. 170 (5), pp: 453-6.

BACKGROUND: Tumours involving the nasal columella have been noted for their aggressive behaviour. Columellar malignancies exhibit perhaps the highest incidence of recurrence and metastasis of all nasal and midfacial cancers of cutaneous origin. Periosteal invasion of maxillary bone and nasal floor may occur 
'barrier free'. Submucosal infiltration of the nasal septum easily develops, leading to potentially lethal invasion of the anterior cranial base. Rapid extension of cancer to the adjacent bony and cartilaginous facial structures requires extensive oncological resections for control. While tumour histology and biologic behaviour remain important prognostic indicators, anatomic location within the columella carries with it a much poorer prognosis than do other nasal cutaneous lesions Recurrent tumours in this location as a result of inadequate initial treatment present a formidable therapeutic challenge. PATIENTS AND METHODS: Fourteen patients who had initially been 'adequately' treated for cutaneous malignancies of the nasal columella were seen from 1982 to 1992 by the authors because of local tumour recurrence. Histologically, four of the 14 were initially basal cell epithelioma, seven were squamous cell epithelioma, one was squamous/basal cell epithelioma, one was melanoma, and one was anaplastic. By the time tumour regrowth was recognized (mean 24 months), 100 per cent demonstrated body involvement of the premaxilla or nasal floor, 57 per cent (eight of 14) nodal metastasis to the parotid or neck, and 43 per cent (six of 14) extension to the anterior skull base. Thirty-six per cent (five of 14) had undergone unsuccessful radiation therapy in an attempt to control the disease. RESULTS: All patients underwent radical oncological surgery with or without postoperative radiation therapy in an attempt to control the tumours, including combinations of anterior skull base resection, partial or total rhinectomy. partial maxillectomy, and parotidectomy with radical neck dissection. At five years, five patients ( 36 per cent) had no evidence of disease and one (seven per cent) was alive with disease; at 54 months, six (43 per cent) had no evidence of disease and one (seven per cent) was alive with disease: at 42 months, seven ( 50 per cent) were alive with no disease. CONCLUSIONS: In this series, an aggressive surgical approach successfully salvaged 43 per cent of recurrences at five years and 50 per cent at 54 months. Although overall prognosis remains poor, radical surgery appears a viable modality for rescue of recurrent malignancies of the columella. In view of the ability to control recurrence, more aggressive initial therapy might improve the prognosis of the early columellar lesion. Author

Prediction of potential metastatic sites in cutaneous head and neck melanoma using Iymphoscintigraphy. O'Brien, C. J., Uren. R. F., Thompson, J. F., Howman-Giles. R. B.. Petersen-Schaefer, K., Shaw, H. M., Quinn, M. J., McCarthy, W. H. Sydney Melanoma Unit, Royal Prince Alfred Hospital, NSW, Australia. American Journal of Surgery (1995) November, Vol. 170 (5), pp: 461-6.

BACKGROUND: The technique of lymphoscintigraphy may allow a more selective approach to the management of clinically negative neck nodes among patients with cutaneous head and neck melanoma. PATIENTS AND METHODS: A group of 97 patients with cutaneous head and neck melanoma had preoperative lymphoscintigraphy using intradermal injections of technetium $99 \mathrm{~m}$ antimony trisulfide colloid to identify sentinel nodes. Fifty-one patients were eligible for clinical analysis after initial definitive treatment by wide excision only $(n=11)$, wide excision and elective dissection of the neck $(n=19)$ or axilla $(n=1)$, or wide excision and a sentinel node biopsy procedure $(n=20)$. RESULTS: Sentinel nodes were identified in 95 of 97 lymphoscintigrams, and 85 per cent of patients had multiple sentinel nodes. In 21 patients ( 22 per cent). sentinel nodes were identified outside the parotid region and the five main neck levels, mostly in postauricular nodes $(n=13)$. Lymphoscintigrams were discordant with clinical predictions in 33 patients ( 34 per cent). Lymph nodes were positive in four elective dissections and four sentinel node biopsies. Among 16 patients evaluable after wide excision and a negative sentinel node biopsy, four patients subsequently developed metastatic nodes; however, confident identification of all nodes marked as sentinel nodes on lymphoscintigraphy was not achieved at the original biopsy procedure in three of these patients CONCLUSIONS: Lymphoscintigraphy and sentinel node biopsy are more difficult to perform in the head and neck than in other parts the body. The reliability of sentinel node biopsy based on lymphoscintigraphy may be improved by identifying and marking all nodes that are considered to receive direct lymphatic drainage from the primary melanoma, and by use of a gamma probe intraoperatively. Author.

The use of the Hayek Oscillator during microlaryngeal surgery.
Monks. P. S., Broomhead, C. J., Dilkes M. G., McKelvie, P. Nuffield Department of Anaesthetics, John Radcliffe Hospital. Headington, Oxford. Anaesthesia (1995) October, Vol. 50 (10) pp: 865-9.

An extremely mounted cuirass ventilator, the Hayek Oscillator, was used on 41 patients undergoing surgery to the larynx without the use of tracheal tube. Gas exchange and cardiovascular parameters remained satisfactory during the use of this technique which offers a significant advance over existing tubeless methods of anaesthesia. Author.

Intralesional laser photocoagulation-steroids as an adjunct to surgery for massive hemangiomas and vascular malformations. Apfelberg, D. B. Atherton Plastic Surgery and Laser Center, CA 94027, USA. Annals of Plastic Surgery (1995) August, Vol. 35 (2). pp: 144-8; discussion 149.

Massive hemangiomas and vascular malformations have been treated with lasers and adjunctive measures. Intralesional laser photocoagulation involves the direct penetration of a tumour by a yttrium-aluminum-garnet laser fibre with photocoagulation at various depths and levels to produce thromboses and controlled coagulation necrosis thus shrinking and blanching the malformation. Seven children have benefited from intralesional laser photocoagulation as well as steroids, embolization, and excisional surgery. Intralesional laser photocoagulation has contributed to the initial shrinkage and fibrosis of these massive vascular tumours in preparation for surgery or as only treatment in some cases. Author.

Audiological assessment of infants and children with preauricular tags. Franccois, M., Wiener-Vacher, S. R., Falala, M., Narcy, P. Department of Otorhinolaryngology, Hopital Robert-Debre. Faculty Bichat, University Paris VII, France. Audiology (1995) January-February, Vol. 34 (1), pp: 1-5.

A previous study found sensorineural hearing loss in 13 per cent of 178 children with solitary preauricular tags. The present results are quite different. Audiological assessment was performed on 42 children (median age of two months) with prearicular tags as the sole anatomical malformation. Tests included behavioural audiometry and transient evoked otoacoustic emissions. Twenty-two subjects were also tested with auditory brainstem recording while under general anesthesia during surgical excision of the tag. None of the children had a sensorineural hearing loss, but four had a bilateral or unilateral conductive hearing loss due to secretory otitis media. It is concluded that solitary ear tags are not a high risk factor for sensorineural hearing loss if not associated with a family history of hearing loss. Author.

Treatment of cutaneous angiosarcomas of the head and neck. Veness. M.. Cooper. S. Department of Radiation Oncology. St Vincent's Hospital, Darlinghurst, New South Wales, Australia. Australasian Radiology (1995) August, Vol. 39 (3), pp. 277-81.

A total of six patients with cutaneous angiosarcomas involving the head and neck were treated during the period 1985-1994. The distribution of sites between the scalp and the rest of the head and neck was equal. Initial local treatment involved surgery for four patients and radiotherapy for two patients. In the surgical group, following seemingly adequate macroscopic surgical excision, all four patients had inadequate surgical margins on histology. Five of six patients had disease recurrence within the head and neck and of these a further three went on to have a second episode of recurrence. Median time to recurrence was eight months. All cases were misdiagnosed clinically at initial presentation and three of six had initial inconclusive histology. Cutaneous angiosarcomas of the head and neck is an aggressive multicentric cancer. Radiotherapy offers the only real chance of obtaining adequate local control even if surgical excision is carried out. Radiotherapy must encompass wide fields and whole scalp irradiation is mandatory for scalp lesions. A combination of laterally opposed photon and electron fields provides a simple, quick and effective means to deliver a tumoricidal dose of $50 \mathrm{~Gy}$ to the whole scalp with minimal irradiation of the underlying brain. Author

Motion sickness incidence during a round-the-world yacht race. Turner, M., Griffin, M. J. Human Factors Research Unit, University of Southampton, England. Aviation Space and Environmental Medicine (1995) September, Vol. 66 (9), pp. 849-56. Motion sickness experiences were obtained from participants in a 
nine month, round the world yacht race. Race participants completed questionnaires on their motion sickness experience one week prior to the start of the race, during the race, and following the race. Yacht headings, sea states, and wind directions were recorded throughout the race. Illness and the occurrence of vomiting were related to the duration at sea and yacht encounter directions relative to the prevailing wind. Individual crewmember characteristics, the use of anti-motion sickness drugs, activity while at sea, and after-effects of yacht motion were also examined with respect to sickness occurrence. Sickness was greatest among females and younger crewmembers, and among crewmembers who used anti-motion sickness drugs. Sickness varied as a function of drug type and activity while at sea. Crewmembers who reported after-effects of yacht motion also reported greater sickness while at sea. The primary determinants of motion sickness were the duration of time spent at sea and yacht encounter direction to the prevailing wind. Author.

\section{A placebo-controlled study of fluticasone propionate aqueous nasal spray and beclomethasone dipropionate in perennial rhinitis: efficacy in allergic and non-allergic perennial rhinitis Scadding, G. K.. Lund, V. J.. Jacques, L. A., Richards, D. H. Royal National Throat, Nose and Ear Hospital. London, UK. Clinical and Experimental Allergy (1995 August, Vol. 25 (8), pp: 737-43. \\ BACKGROUND: Fluticasone propionate is a new potent, topically active corticosteroid with negligible oral bioavailability. Data on its comparative efficacy in perennial allergic and non- allergic rhinitis are limited. OBJECTIVE: To compare the efficacy and safety of fluticasone propionate aqueous nasal spray (FPANS) 200 micrograms once or twice daily with beclomethasone dipropionate aqueous nasal spray (BPD) 200 micrograms twice daily and placebo in patients with allergic and non-allergic perennial rhinitis METHODS: The 12-week study had a multi- centre, double-blind, randomized, parallel group design. Efficacy was assessed from symptom scores recorded on daily diary cards. RESULTS: FPANS 200 micrograms once or twice daily was significantly better than placebo but not better than BDP in relieving the nasal symptoms of rhinitis. FPANS at either dose was equally effective in the treatment of allergic and non-allergic perennial rhinitis. There were few adverse events and no treatment-related abnormalities in laboratory measurements in either FPANS-treated group. Comparison between treatment groups indicated that FPANS was as well tolerated as placebo and BDP at the doses studied. CONCLUSIONS: In the majority of patients FPANS 200 micrograms once daily in as effective as BDP 200 micrograms twice daily in the relief of perennial allergic rhinitis Author.}

The use of ultrasound-guided cutting-needle biopsy in the neck. Bearcroft, P. W., Berman. L. H.. Grant, J. Department of Radiology, Addenbrooke's Hospital NHS Trust, Cambridge, UK Clinical Radiology (1995) October, Vol. 50 (10), pp: 690-5.

Histological analysis of an abnormal neck mass has traditionally required an excision biopsy under general anaesthetic. We evaluated the safety and accuracy of a new spring loaded cutting-needle for obtaining tissues cores of neck masses under ultrasound (US) guidance. Sixty biopsies were performed on an outpatient basis under local anaesthesia in 52 patients referred with a neck mass. Patients ranged in age from 10 months to 89 years and masses varied in size from $5 \mathrm{~mm}$ to $5 \mathrm{~cm}$. Fifty-eight of the 60 biopsies provided a diagnostic histological specimen. Of the remaining two patients, one required open biopsy and the other returned for a second needle biopsy. All five cases of lymphoma were correctly diagnosed on needle biopsy; in three cases full tumour sub-classification was possible, but in two patients an open biopsy was subsequently required. In 49 patients the needle biopsy obviated the need for a surgical biopsy for diagnostic purposes although in 12 cases a therapeutic excision biopsy was performed Apart from one subclinical haematoma, visualized on U.S., there were no immediate or delayed complications. In all patients. the histological diagnosis was compatible with subsequent clinical, radiological, surgical or autopsy findings. Cutting-needle biopsy of neck masses under U.S. guidance is an effective and safe procedure which should be considered before resorting to routine surgery. Author.

Acoustic parameters of nasalized vowels in hearing-impaired and normal-hearing speakers. Chen, M. Y. Department of Electrical
Engineering and Computer Science, Massachusetts Institute of Technology, Cambridge 02139, USA. Journal of the Acoustical Society of America (1995) November, Vol. 98 (5 Pt 1), pp: 2443-53. One of the more prevalent abnormalities that contribute to reduced intelligibility in the speech of hearing-impaired speakers is inadvertent nasalization. Acoustic analysis of the speech of hearing-impaired children and nasalized vowels of normal-hearing adults indicate the presence of an extra pole-zero pair between the first and second formants and a reduced first-format prominence. The difference between the amplitude of the first formant and the amplitude of the extra peak, A1-P1, was shown to be a promising measure that correlates with listener judgments of the degree of vowel nasality in utterances of hearing-impaired and normalhearing children. To obtain further validation of these parameters as measures of nasality, A1 and P1 were systematically manipulated in synthetic utterances. The results of perceptual experiments with a number of synthesized words (of the form bVt) showed somewhat different relative importance of the two parameters, depending on the vowel. High correlation of A1-P1 with the average nasality perception judgments was found for each of the stimuli except those for which the first formant was very close to the extra peak frequency. A procedure was developed for correcting the value of A1-P1 to normalize for different vowels, based on the frequencies of the first two formants. Author.

Influenza A virus-induced acute otitis media. Buchman, C. A. Doyle, W. J., Skoner, D. P., Post, J. C., Alper, C. M., Seroky, J. T., Anderson, K.. Preston, R. A., Hayden, F. G., Fireman, P., et al. Department of Otolaryngology, University of Pittsburgh School of Medicine, Pennsylvania, USA. Journal of Infectious Diseases (1995) November, Vol. 172 (5), pp: 1348-51.

To better understand the significance of viral upper respiratory tract infections in the pathogenesis of acute otitis media (OM), 27 adults underwent intranasal inoculation with influenza $A$ virus. Monitoring consisted of antibody titre determination, tympanometry, and otoscopy. Microbiologic analysis consisted of cultures and polymerase chain reaction (PCR)-based detection for influenza A virus. Streptococcus pneumoniae, Haemophilus influenzae, and Moraxella catarrhalis. All subjects became infected with the challenge virus. By day 4, 16 (59 per cent) developed middle ear pressures of $-100 \mathrm{~mm} \mathrm{H} 20$ or below and four ( 25 per cent) of them developed OM. One subject (four per cent) developed purulent $\mathrm{OM}$ requiring myringotomy for pain relief. Middle ear effusion cultures were negative. PCR analysis of that subject's middle ear effusion and nasal washes were positive for influenza $\mathrm{A}$ virus and $S$. pneumoniae. These findings support a causal role for viral upper respiratory tract infections in the pathogenesis of $\mathrm{OM}$, possibly mediated by middle ear underpressures and viral and bacterial middle ear infection. Author.

Concurrent trigeminal, abducens and facial nerve palsies presenting as false localizing signs: case report. Ro, L. S., Chen, S. T., Tang, L. M., Wei, K. C. Department of Neurology, Chang Gung Memorial Hospital and Medical College. Taipei, Taiwan. Neurosurgery (1995) August, Vol. 37 (2), pp: 322-4; discussion 324-5. Multiple cranial nerve dysfunction presenting as false localizing signs is rare. We report a 20 -year-old woman who had concurrent trigeminal sensory disturbance, abducens, and peripheral facial nerve palsies in association with a contralateral acoustic neuroma. After surgery, the trigeminal nerve disturbance and the abducens nerve palsy completely recovered, but the peripheral facial nerve palsy persisted. The nature of tumour, the presence of brain stem distortion, the anatomic variation of posterior fossa, and the relationships of cranial nerves and nearby blood vessels, which are factors influencing the occurrence of false localizing, are briefly discussed. Author.

A pacifier increases the risk of recurrent acute otitis media in children in day care centres. Niemela, M., Uhari M., Mottonen, M. Department of Pediatrics, University of Oulu, Finland. Pediatrics (1995) November, Vol. 96 (5 Pt 1), pp: 884-8.

OBJECTIVE: To follow-up a previous retrospective analysis in which we found the use of a pacifier to be a risk factor for recurrent acute otitis media (AOM). METHOD: In the present prospective study, the occurrence of AOM and the use of a pacifier were recorded in 845 children attending day care centres during a 15-month period. RESULTS: More than three attacks of AOM occurred in 29.5 per cent of the children younger than two years 
using pacifiers and in 20.6 per cent of those not doing so (relative risk, 1.6; 95 per cent confidence interval $(\mathrm{CI}), 0.6,4.1$ ); in children two to three years of age, the figures were 30.6 per cent and 13.2 per cent, respectively (relative risk, $2.9 ; 95$ per cent $C I, 1.2,7.3$ ). Logistic modelling with adjustment for age and the duration of monitoring showed the occurrence of AOM to be associated with the time during which a pacifier was used. The use of a pacifier increased the annual incidence of AOM from 3.6 (95 per cent $\mathrm{CI}$, $2.5,4.9)$ to 5.4 episodes $(4.4,6.6)$ in children younger than two years and from $1.9(1.4,2.5)$ to $2.7(2.2,3.3)$ in children two to three years of age. The population-attributable risk of AOM attacks due to the use of a pacifier was 176 attacks, i.e. 459 to 635 attacks per year, in the youngest children and 69 attacks, i.e. from 264 to 333 attacks per year, in those two to three years of age. It can be calculated that the use of a pacifier was responsible for 25 per cent of the attacks in children younger than three years. Breastfeeding, parental smoking, thumb sucking, using a nursing bottle, and the social class of the family failed to show such strong associations with the occurrence of AOM. CONCLUSION: We conclude that the use of a pacifier is a significant risk factor for recurrent AOM and suggest that pacifiers should be used only during the first 10 months of life, when need for sucking is strongest, and AOM is uncommon. Author.

Diagnosis and treatment of ear disease among children in the Ellisras district. An outreach programme. van Rooy, C. H., Swart, J. G., Op t-Hof, J., Vlantis A. C., Ahmed, M. R., Venter, P. H. Department of Otorhinolaryngology, H.F. Verwoerd Hospital. South African Medical Journal (1995) July, Vol. 85 (7), pp: 675-7. Audiologists and ENT registrars examined 2,036 children aged 10 years or younger by means of pure-tone audiometry, tympanometry and otoscopic examinations. Twenty per cent of these children had ear pathology and 7.5 per cent had impaired hearing. Forty-three pus swabs taken from patients with suppurative otitis media most often cultured Proteus mirabilis and Pseudomonas species. The prevalence of otitis media with effusion was twice as high in white children as in black children, resulting in a much higher prevalence of hearing loss in the first group. The black children had seven times more dry perforations of the tympanic membrane and 11 times more wet perforations. A hundred and thirty-six patients were treated by the team and 137 were referred for further treatment. Sixteen operations were performed at a later stage. Further programmes are needed to diagnose and treat ear disease in rural communities Author.

Phenotypic manifestations of branchio-oto-renal syndrome. Chen, A., Francis, M., Ni, L., Cremers, C. W., Kimberling W. J., Sato, Y., Phelps, P. D., Bellman, S. C., Wagner, M. J., Pembrey, M., et al. Department of Otolaryngology - Head and Neck Surgery, University of Iowa, Iowa City 52242, USA. American Journal of Medical Genetics (1995) September 25, Vol. 58 (4), pp: 365-70. Branchiootorenal (BOR) syndrome is a variable, autosomaldominant disorder of the first and second embryonic branchial arches, kidneys, and urinary tract. We describe the phenotype in 45 individuals highlighting differences and similarities reported in other studies. Characteristic temporal bone findings include cochlear hypoplasia (four/five of normal size with only two turns), dilation of the vestibular aqueduct, bulbous internal auditory canals, deep posterior fossae, and acutely-angled promontories Author.

Acute otitis media in children: a study of nasopharyngeal carriage of potential pathogens and therapeutic efficacy of cefixime and amoxicillin-clavulanate. Boulesteix, J., Begue, P., Dubreuil, C., Megraud. F., Dabernat, H., Geslin, P., de La Rocque, F., Trinh, A. Service de Pediatrie II, Centre Hospitalier Regional et Universitaire de Limoges. Infection (1995), Vol. 23 Suppl 2, pp: S79-82. We conducted a large, multicentre, randomized, open-label study throughout France comparing the efficacy and safety of cefixime suspension $(8 \mathrm{mg} / \mathrm{kg} /$ day, b.i.d., for 10 days) versus amoxicillinclavulanate suspension $(80 \mathrm{mg} / \mathrm{kg} /$ day, t.i.d., for 10 days $)$ in 510 children (ages six to 36 months) with acute otitis media. The most frequent microorganisms colonizing the nasopharynx at the start of treatment were Streptococcus pneumoniae (51.5 per cent), Haemophilus influenzae (45 per cent) and Moraxella catarrhalis (30.2 per cent). Rates of beta-lactamase positivity were 32.1 per cent and 95.3 per cent for $H$. influenzae and $M$. catarrhalis, respectvely. Decreased susceptibility of $S$. pneumoniae to penicillin was found in 39.7 per cent of isolates. Clinical efficacy was 87.8 per cent (223/254) for cefixime and 87 per cent (215/247) for amoxicillin-davulanate. At the five-week follow-up visit, relapse had occurred in 15.7 per cent (31/197) of cefixime-treated patients and in 15.6 per cent (32/205) of those treated with amoxicillinclavulanate. We conclude that these two regimens are equally effective in acute otitis media in children. Author.

Association of Epstein-Barr virus with sinonasal angiocentric $\mathbf{T}$ cell Iymphoma. O'Leary, G., Kennedy, S. M. Department of Pathology, Research Foundation, Royal Victoria Eye and Ear Hospital, Dublin, Ireland. Journal of Clinical Pathology (1995) October, Vol. 48 (10), pp: 946-9.

AIM: To investigate whether non-Hodgkin's lymphomas arising in the sinonasal region or Waldeyer's ring contain the Epstein-Barr virus (EBV) genome in lesional tissue. METHOD: Sections from paraffin wax blocks of 22 lymphoid proliferations arising in the sinonasal region or Waldeyer's ring were studied with EBV encoded RNAs (EBER-1 and -2) using in situ hybridization RESULTS: EBV was detected in nuclei of tumour cells of five of seven $T$ cell lymphomas and in nuclei of two of seven diffuse, large cell immunoblastic lymphomas of B phenotype in the sinonasal region. Of tumours arising in Waldeyer's ring, two of 10 nonHodgkin's lymphomas (both large cell) were positive, as was a single case of Hodgkin's disease. Lymphoma of other types including Western type Burkitt's lymphoma, and nodular and diffuse small cleaved cell lymphoma, were negative. CONCLUSION: EBV is highly associated with large cell lymphomas especially $\mathrm{T}$ cell lymphomas of sinonasal origin in the indigenous Irish population, underlining the importance of this virus in nasopharyngeal lymphomas in Northern European as well as Asian populations. Author.

Experimental evaluation and clinical use in the head and neck of a 3M/Precise microvascular anastomotic device. Borja-Morant, A. Navarro-Vila, C., Cuesta-Gil, M., Martin-Sastre, R. General Hospital Gregorio Maranon, Maxillofacial Surgery Department. Madrid, Spain. Journal of Craniomaxillofacial Surgery (1995) October, Vol. 23 (5), pp: 305-11.

Microvascular reconstructions in the head and neck are usually long operating time procedures Mechanical anastomotic devices help to reduce operating time and can reduce anastomotic failures avoiding foreign bodies in the lumen of the vessel. One of these systems is the $3 \mathrm{M} /$ Precise microvascular anastomotic device, it is a non-absorbable device, however, criticisms of this system have been directed to the fact that pulsation of the vessel wall against a rigid structure could lead to thinning of the vessel wall and aneurysm formation. No aneurysms have been found previously in other experimental models Our experimental study on the aorta and vena cava of the rat comprises 25 arterial and 25 venous anastomoses In the arteries, four proximal aneurysms were found. two of these were failures In the venous anastomoses, no failures were found nor aneurysm formation. The system is very useful for performing clinical end to end venous anastomosis helping to reduce anastomotic failures Aneurysms have been found in arteries although four different ring sizes were available. The device is less easy to use in them than in veins and sometimes can be difficult to apply, making manual suturing a better choice for clinical arterial anastomosis. Author.

Virtual image guided navigation in tumour surgery-technical innovation. Wagner, A., Ploder, O., Enislidis G., Truppe, M. Ewers, R. University-Clinic for Maxillofacial Surgery, Vienna, Austria. Journal of Craniomaxillofacial Surgery (1995) October. Vol. 23 (5), pp: 217-3.

We present a new visualization system for image-guided stereotactic navigation in tumour surgery. The combination of frameless stereotactic localization technology with real-time video processing permits the visualization of medical imaging data as a video overlay during the actual surgical procedure. Virtual computer-generated anatomical structures are displayed intraoperatively in a semiimmersive head-up display. This results in surgical navigation assistance without limiting the judgment of the physician based on the continuous observation of the operating field. The case presented documents the potential of augmented reality visualization concepts in tumour surgery of the head. Author.

Antibody-secreting cells and their relation to humoral antibodies 
in serum and in nasopharyngeal aspirates in children with pneumococcal acute otitis media. Nieminen, T., Virolainen, A. Kayhty, H., Jero, J., Karma, P., Leinonen, M., Eskola, J. National Public Health Institute, University of Helsinki, Finland. Journal of Infectious Diseases (1996) January, Vol. 173 (1), pp: 136-41.

Mucosal and systemic antibody responses to pneumococcal capsular polysaccharide were studied in 17 children with cultureverified pneumocoocal acute otitis media. Serotype-specific antibody-secreting cells (ASCs) in peripheral blood and antibodies in acute and convalescent sera and nasopharyngeal aspirates were measured. A polysaccharide-specific ASC response was induced in all subjects. The response was age dependent, and the dominant antibody class was IgA. Three children $>24$ months old had $>100$ IgA-class ASCs/10 (6) cells and serum and nasopharyngeal IgA responses; two had only a nasopharyngeal IgA response. None of eight children $<24$ months old showed a systemic response; however, a nasopharyngeal IgA response was detected in one Results suggest that whole pneumocooci can induce a mucosal polysaccharide-specific antibody response independent of the systemic response. Results are also in accordance with earlier studies suggesting that the mucosal immune system matures earlier in life than does the systemic immune response. Author.

Visual vertigo syndrome: clinical and posturography findings. Bronstein, A. M. MRC Human Movement and Balance Unit Institute of Neurology, National Hospital for Neurology and Neurosurgery, London, UK. Journal of Neurology, Neurosurgery and Psychiatry (1995) November, Vol. 59 (5), pp: 472-6.

Neuro-otological and posturography findings in 15 patients with visually induced vertiginous symptoms (visual vertigo) are reported. Thirteen patients were considered to have a peripheral vestibular disorder; seven had abnormal caloric or rotational test results. Two patients had CNS disorder-a cerebellar degeneration and a brainstem stroke. Posturography testing showed that five patients showed abnormally large body sway induced by full field visual motion stimulation. This group included the two patients with CNS disease and four with strabismic symptoms (diplopia, squint surgery, and ocular muscle weakness). It is concluded that visual vertigo is a heterogeneous syndrome with peripheral or central aetiologies and may occur if patients with balance disorders show high visual field dependence. In patients with visual vertigo, the presence of additional CNS or strabismic symptoms may cause inappropriate postural reactions in environments with conflicting or disorienting visual stimuli, probably by reducing the ability to resolve the sensory conflict. Author.

Laryngeal carcinoma after radiation therapy: correlation of abnormal MR imaging signal patterns in laryngeal cartilage with the risk of recurrence. Castelijns, J. A., van den Brekel, M. W., Tobi, H., Smit, E. M., Golding, R. P., van Schaik, C., Snow, G. B. Department of Radiology, Vrije Universiteit Hospital, Amsterdam, The Netherlands. Radiology (1996) January, Vol. 198 (1), pp: 151-5.

PURPOSE: To correlate abnormal magnetic resonance (MR) imaging signal patterns in cartilage with the effectiveness of radiation treatment. MATERIALS AND METHODS: Eighty previously untreated patients underwent MR imaging and radiation therapy with a curative intent. Cartilage was considered to have an abnormal signal pattern if it had intermediate signal intensity on T-weighted spin-echo (SE) MR images and high signal intensity on T2-weighted SE MR images. The minimum follow-up was two years. RESULTS: Abnormal MR imaging signal patterns of the thyroid cartilage $(p<0.001 ; p<0.04)$ were more ominous than those of other cartilage. Abnormal signal patterns in cartilage of patients with small tumours $\left(<5 \mathrm{~cm}^{3}\right.$ and especially $\left.<1 \mathrm{~cm}^{3}\right)$ were less significant. Abnormal signal patterns in cartilage combined with a large tumour $\left(>5 \mathrm{~cm}^{3}\right)$ worsened the prognosis significantly $(p<0.05)$. CONCLUSION: Abnormal MR imaging signal patterns in cartilage may not indicate a poor prognosis in every case. Abnormal signal intensity in the thyroid cartilage combined with a tumour volume of $>5 \mathrm{~cm}^{3}$, however, appears to indicate an adverse prognosis with regard to tumour recurrence. Author. 